\title{
The problematic backreaction of SUSY-breaking
}

\section{branes}

\section{Johan Blåbäck, ${ }^{a}$ Ulf H. Danielsson, ${ }^{a}$ Daniel Junghans, ${ }^{b}$ Thomas Van Riet, ${ }^{a}$} Timm Wrase ${ }^{c}$ and Marco Zagermann ${ }^{b}$

${ }^{a}$ Institutionen för Fysik och Astronomi, Uppsala Universitet, Box 803, SE-751 08 Uppsala, Sweden

${ }^{b}$ Institut für Theoretische Physik $\&$ Center for Quantum Engineering and Spacetime Research, Leibniz Universität Hannover, Appelstraße 2, $3016^{r} 7$ Hannover, Germany

${ }^{c}$ Department of Physics, Cornell University, Ithaca, NY 14853, U.S.A.

E-mail: johan.blaback@fysast.uu.se, ulf.danielsson@fysast.uu.se, daniel.junghans@itp.uni-hannover .de, thomas.vanriet@fysast .uu . se, timm.wrase@cornell.edu, marco.zagermann@itp.uni-hannover.de

ABSTRACT: In this paper we investigate the localisation of SUSY-breaking branes which, in the smeared approximation, support specific non-BPS vacua. We show, for a wide class of boundary conditions, that there is no flux vacuum when the branes are described by a genuine delta-function. Even more, we find that the smeared solution is the unique solution with a regular brane profile. Our setup consists of a non-BPS $\mathrm{AdS}_{7}$ solution in massive IIA supergravity with smeared anti- $D 6$-branes and fluxes T-dual to ISD fluxes in IIB supergravity.

KEYwORDS: Flux compactifications, Superstring Vacua, D-branes, dS vacua in string theory 


\section{Contents}

1 Introduction 1

2 A simple non-BPS setup on $\mathrm{AdS}_{7} \times S^{3} \quad 3$

2.1 Type IIA supergravity and smeared $D 6 / \overline{D 6}$-branes 4

2.2 The smeared solution 5

3 Perturbative stability $\quad 5$

3.1 The left-invariant closed string moduli 5

$\begin{array}{lll}3.2 & \text { Open string moduli } & 7\end{array}$

4 Localisation $\quad 8$

4.1 The ansatz 8

$\begin{array}{lll}4.2 & \text { Delta-function sources } & 10\end{array}$

$\begin{array}{lll}4.3 & \text { Regularised sources } & 14\end{array}$

$\begin{array}{ll}\text { 4.4 Summary of results } & 16\end{array}$

$\begin{array}{lll}5 & \text { Discussion } & 17\end{array}$

A Smeared non-BPS solutions for general $d$ and $\eta \quad 19$

$\begin{array}{lll}\text { A.1 The ansatz } & 19\end{array}$

$\begin{array}{ll}\text { A.2 The solutions with } \eta= \pm 1 & 20\end{array}$

$\begin{array}{lll}\text { A.3 The solutions with general } \eta & 21\end{array}$

\section{Introduction}

$D$-branes and orientifold planes are necessary ingredients in semi-realistic compactifications of type II string theories, where they play an important role for the implementation of chiral matter, supersymmetry breaking or tadpole cancelation in the presence of fluxes. Being massive and charged localised objects, they generally also backreact on the geometry and source a non-trivial profile for the warpfactor, dilaton and some of the RR-potentials. Apart from the special cases where this backreaction is absent due to a local cancelation of charge and tension for suitably stacked $D$-branes and $O$-planes, one needs to take into account these backreaction effects, or make sure they can be neglected.

The GKP setup [1] and its T-duality relatives (see e.g. [2, 3]) are the few examples where such backreaction effects are quite well understood, ${ }^{1}$ but much less is known about more general cases, e.g. with intersecting branes or cases for which the sources and fluxes are not all mutually BPS as in de Sitter vacua.

\footnotetext{
${ }^{1}$ See $[4,5]$ for fully backreacted solutions of codimension-2 branes in $6 \mathrm{D}$ supergravity.
} 
A common approach to deal with the effects of localised sources is to take them into account only in an averaged or integrated sense. At the level of the 10D field equations, this procedure corresponds to "smearing" the $D$-branes and $O$-planes in the directions perpendicular to their world volumes; ${ }^{2}$ in other words, one replaces the delta-function of the energy and charge density along the transverse brane coordinates by a constant in the equations of motion,

$$
\delta \rightarrow \text { const. }
$$

On a torus, the smearing could be viewed as a truncation of the Fourier expansion of the delta-function to the constant term. For compactifications on more general group or coset manifolds (including the much studied examples of spheres and (twisted) tori), this smearing of the sources allows a consistent truncation of the effective lower-dimensional theory to the sector of left-invariant modes $[2,7,8]$. It is this consistent truncation property that is usually (albeit often implicitly) exploited in the derivation of effective gauged supergravity theories, see e.g. [9] and references therein. At the level of the 10D field equations, the smearing in general leads to substantial simplifications regarding the profile of the warp factor, the dilaton and some RR-potentials and allows the construction of many explicit 10D solutions, see e.g. [10, 11].

As the smearing of a brane-like source is a drastic modification of the original setup, one should wonder to what extent this can actually be a good approximation to a solution with truly localised branes. More precisely, there are two questions one may ask in this context:

1. Does a smeared solution always imply a localised solution, or could there be smeared solutions that have no well-defined localised counterpart?

2. In case a smeared solution does have a localised version, how physically different are these two solutions, e.g. regarding the vacuum expectation values or masses of stabilised moduli or the value of the effective cosmological constant?

In this paper we want to address the first question, ${ }^{3}$ building upon our previous work [3]. There we studied this question for BPS-type solutions that generalize the GKP solutions [1] to various spacetime dimensions and found that the BPSness played a crucial role in achieving successful localisation. Intuitively, this may be understood as a consequence of the cancelation of forces between the fluxes and the localised sources in such setups. By BPS-type solutions we mean that the solutions satisfy a Bogomol'nyi bound, but are not necessarily supersymmetric. As an example, the GKP solutions [1] are BPS but only supersymmetric when the ISD flux is of the specific complexity type $(2,1)$ (see also [19]).

For non-BPS solutions, on the other hand, it becomes unclear whether localisation works and simple arguments presented in [3] indicate that it may not work in general. In this paper we further improve on the arguments given in [3] and consider a particular setup

\footnotetext{
${ }^{2}$ We refer to [6] for a proper treatment of smeared sources in flux compactifications.

${ }^{3}$ The second question is what "warped effective field theory" is concerned with, see e.g. [12-18] and references therein.
} 
where the effects of the brane localisation can be traced explicitly. Concretely, we consider spacetime-filling (anti-)D6-branes on $\mathrm{AdS}_{7} \times S^{3},{ }^{4}$ for which a solution exists in the limit of smeared sources [3]. This example is closely related to the (smeared) Minkowski solutions of GKP [1] and their generalisations to different dimensions [3] and configurations with mixed sources (cf. appendix A). We show that this solution is perturbatively stable in the leftinvariant closed string modes, which is the sector usually considered in lower-dimensional supergravity analyses.

Next we study the localisation of the branes. We start with considering the branes to be fully localised as delta-functions. Under the assumption that the fluxes very close to the sources are mutually BPS with the source, we find that the equations of motion do not allow for any possibility to match the behaviour of the fields, close to and away from the sources. Therefore it is not possible to construct fully localised solutions with this BPSlike behaviour near the branes. We point out a caveat in the case that one uses boundary conditions for which the fluxes near the sources are not mutually BPS with the sources. However, it is unclear how such boundary conditions could be consistent with a static solution, since there should be a non-zero force between the fluxes and the sources. We will present a detailed discussion of boundary conditions at the sources and a generalisation of our argument to different setups in [20].

In this paper we then present, instead, an independent calculation that supports the above conclusions. In order to do so, we consider regularised brane profiles that may approximate the previously considered delta-type profiles to arbitrary precision. If we assume a smooth profile, we find a one parameter family of solutions which correspond to exactly the one parameter family of coordinate transformations of the fully smeared solution. This means that there exists no solutions with regular brane profiles other than the fully smeared one. This is in contrast to the BPS solutions given by the GKP solutions and their T-duals, since they solve the equations of motion for any brane profile. We then use this to rule out also regularised brane profiles with the shape of step functions.

In the discussion we emphasise the possible implications of our results on known solutions that are utilising the smeared approximation of $D$-branes or $O$-planes.

\section{$2 \quad$ A simple non-BPS setup on $\mathrm{AdS}_{7} \times S^{3}$}

In this section we present a simple non-BPS setup on $\mathrm{AdS}_{7} \times S^{3}$ with spacetime-filling (anti-)D6-branes as sources. This setup yields a smeared solution and allows to explicitly tackle the questions raised in the introduction.

We should point out that this example is a special case of a more general class of setups for which smeared solutions exist (see appendix A). These setups have $d$-dimensional AdS, Minkowski or dS spacetimes and a mixture of spacetime filling sources that allows net charge/tension ratios that can be non-extremal, $T=\eta Q$ with arbitrary $\eta$. Those solutions

\footnotetext{
${ }^{4}$ The sphere geometry is not necessary for the smeared solution as any positively curved Einstein space could fulfill the smeared equations of motion. The sphere is chosen so as to make a fully explicit discussion of the localised case feasible.
} 
with $|\eta|=1$ were already discussed in [3]. The specific $\operatorname{AdS}_{4}$ solution with $|\eta|=1$ and $S^{3} \times S^{3}$ as internal space was first established in [21].

In the following, we shall restrict our discussion to the $\mathrm{AdS}_{7}$ example with $|Q|=T>0$, since it is the simplest case of this class of models and we consider it sufficient for supporting the main point of this paper, namely that localisation of smeared non-BPS solutions may fail.

\subsection{Type IIA supergravity and smeared $D 6 / \overline{D 6}$-branes}

To establish our notation and conventions we present the equations of motion for type IIA supergravity with spacetime-filling $D 6 / \overline{D 6}$-branes in Einstein frame. We use the same conventions as in [3]. ${ }^{5}$ Throughout the paper $a, b$ are 10D indices, $\mu, \nu$ are external and $i, j$ are internal indices. The bosonic sector of type IIA supergravity contains the metric $g_{a b}$, the dilaton $\phi$, the $H$ field strength, as well as the RR field strengths $F_{0}, F_{2}, F_{4}$. Since we compactify to $\mathrm{AdS}_{7}$, however, we do not consider any external fields that break translational invariance and hence do not turn on $F_{4}$.

The trace reversed Einstein equation is then

$$
\begin{aligned}
R_{a b}= & \frac{1}{2} \partial_{a} \phi \partial_{b} \phi+\frac{1}{2} \mathrm{e}^{-\phi}|H|_{a b}^{2}-\frac{1}{8} \mathrm{e}^{-\phi} g_{a b}|H|^{2}+\frac{1}{16} \mathrm{e}^{\frac{5}{2} \phi} g_{a b} F_{0}^{2} \\
& +\frac{1}{2} \mathrm{e}^{\frac{3}{2} \phi}\left|F_{2}\right|_{a b}^{2}-\frac{1}{16} \mathrm{e}^{\frac{3}{2} \phi} g_{a b}\left|F_{2}\right|^{2}+\frac{1}{2}\left(T_{a b}^{\mathrm{loc}}-\frac{1}{8} g_{a b} T^{\mathrm{loc}}\right),
\end{aligned}
$$

where $|A|_{a b}^{2} \equiv \frac{1}{(p-1) !} A_{a a_{2} \ldots a_{p}} A_{b}^{a_{2} \ldots a_{p}},|A|^{2} \equiv \frac{1}{p !} A_{a_{1} \ldots a_{p}} A^{a_{1} \ldots a_{p}}$.

The non-vanishing part of the local stress tensor is given by

$$
T_{\mu \nu}^{\mathrm{loc}}=-\mathrm{e}^{\frac{3}{4} \phi} \mu_{6} g_{\mu \nu} \delta(D 6),
$$

where $\mu_{6}$ is a positive number, and $\delta(D 6)$ is the delta distribution with support on the $D 6$-brane world volume(s), i.e. it may implicitly also include sums of parallel $D 6$-branes. The dilaton equation of motion is given by

$$
\nabla^{2} \phi=-\frac{1}{2} \mathrm{e}^{-\phi}|H|^{2}+\frac{5}{4} \mathrm{e}^{\frac{5}{2} \phi} F_{0}^{2}+\frac{3}{4} \mathrm{e}^{\frac{3}{2} \phi}\left|F_{2}\right|^{2}+\frac{3}{4} \mathrm{e}^{\frac{3}{4} \phi} \mu_{6} \delta(D 6) .
$$

The Bianchi identities for the field strengths are

$$
\mathrm{d} H=0, \quad \mathrm{~d} F_{0}=0, \quad \mathrm{~d} F_{2}=H F_{0} \pm \mu_{6} \delta_{3},
$$

where the upper sign of the source term is for D6-branes and the lower sign for anti$D 6$-branes. $\delta_{3}$ is shorthand for the normalized volume 3 -form transverse to the $D 6$-brane multiplied by $\delta(D 6), \delta_{3}=\delta(D 6) \star_{3} 1$.

$$
\int \delta(D 6) \star_{3} 1=1
$$

The equations of motion for $H$ and $F_{2}$ read

$$
\mathrm{d}\left(\mathrm{e}^{-\phi} \star H\right)=-\mathrm{e}^{\frac{3}{2} \phi} \star F_{2} \wedge F_{0}, \quad \mathrm{~d}\left(\mathrm{e}^{\frac{3}{2} \phi} \star F_{2}\right)=0 .
$$

\footnotetext{
${ }^{5}$ They are related to the conventions of [22] by going to Einstein frame and changing the sign of $H$.
} 
In the smeared limit we have

$$
\delta(D 6) \rightarrow \frac{1}{V},
$$

where $V$ is the volume of the space transverse to the branes.

\subsection{The smeared solution}

In the smeared limit, we can set $F_{2}=0$ and assume that all other fields are constant. We furthermore assume that the metric

$$
\mathrm{d} s_{10}^{2}=\mathcal{R}_{\mathrm{AdS}}^{2} \mathrm{~d} s_{7}^{2}+\mathcal{R}_{S^{3}}^{2} \mathrm{~d} s_{3}^{2},
$$

is a direct product $\mathrm{AdS}_{7} \times S^{3}$ with $\mathcal{R}_{\text {AdS }}$ the AdS radius and $\mathcal{R}_{S^{3}}$ the radius of the threesphere and write

$$
H=\lambda F_{0} \mathrm{e}^{\frac{7}{4} \phi} \star_{3} 1
$$

Going through the equations of motions (2.1), (2.3), (2.4), (2.6), one then finds that all are solved for

$$
\mathcal{R}_{S^{3}}^{2}=\frac{\mathcal{R}_{\mathrm{AdS}}^{2}}{12}=\frac{7^{7 / 8}}{\sqrt{2} 5^{5 / 4}}\left(F_{0}^{2} h^{5}\right)^{1 / 4}, \quad \mathrm{e}^{\phi}=\sqrt{\frac{20}{7 \sqrt{7}}} \frac{1}{\sqrt{F_{0}^{2} h}}, \quad \lambda={ }_{(+)} \frac{5}{2}, \quad \mu_{6}=2\left|F_{0}\right| h \pi^{2},
$$

where $h=\sqrt{|H|^{2} \mathcal{R}_{S^{3}}^{6}}$ is independent of $\mathcal{R}_{S^{3}}$. The (lower) upper sign in $\lambda$ is for (anti-)D6branes. For large flux parameters, we thus have large volumes and small string coupling.

\section{Perturbative stability}

In this section we want to verify the perturbative stability of the smeared solution of the previous section. For practical reasons, we do this only for the subsector of the leftinvariant modes. This is precisely the sector that underlies the usual gauged supergravity description. In case there happen to be tachyonic modes among the higher Kaluza-Klein modes or in the sector that is not left-invariant, they would hence also be missed in the gauged supergravity approach, which is the standard tool to find new vacua.

We first consider the closed string sector and perform the computation in all details. For the open string sector we are necessarily sketchy and point out where possible instabilities could reside, if any.

\subsection{The left-invariant closed string moduli}

When we regard the three-sphere as the group manifold $\mathrm{SU}(2)$ there is a standard way to take into account a subset of the degrees of freedom, namely the left-invariant modes [23]. The left-invariant modes are the lower-dimensional fields that one obtains by expanding the supergravity fields in the left-invariant Maurer-Cartan basis of SU(2). These forms form a global coframe on the group manifold and obey the characteristic equation

$$
\mathrm{d} e^{i}=-\frac{1}{2} f_{j k}^{i} e^{j} \wedge e^{k} .
$$


We take a basis for the structure constants for which [24]

$$
f_{j k}^{i}=q \epsilon_{j k l} \delta^{l i}
$$

where $q$ is a number that gives the curvature scale.

There are six metric scalars, three scalars from $C_{1}$, three from $B_{2}$, one from $C_{3}$ and one being the dilaton $\phi$ in ten dimensions. This adds up to 14 scalars that form the coset manifold $\mathrm{SL}(5) / \mathrm{SO}(5)$. Of course when we add the "open string degrees" of freedom, coming from the $D 6 / \overline{D 6}$-branes, we have more scalars, and the coset enlarges.

Let us write the moduli explicitly in a convenient basis, by which we mean a field basis for which the field metric, at the solution, is canonically normalised. This then allows us to compute the scalar masses by simply diagonalising $\partial_{i} \partial_{j} V$.

The 10D metric, in Einstein frame, is written as follows

$$
\mathrm{d} s_{10}^{2}=\mathrm{e}^{2 \alpha v} \mathrm{~d} s_{7}^{2}+\mathrm{e}^{2 \beta v} \mathrm{~d} s_{3}^{2},
$$

where $v$ is the volume modulus and the numbers $\alpha$ and $\beta$ are chosen such that the reduced theory is in Einstein frame and that the volume modulus is canonically normalised

$$
\alpha^{2}=\frac{3}{80}, \quad \beta=-\frac{5}{3} \alpha
$$

The internal metric is then written as

$$
\mathrm{d} s_{3}^{2}=M_{i j} e^{i} \otimes e^{j},
$$

where $M$ is the symmetric, positive definite, metric-moduli matrix. It is explicitly given by $M=L L^{T}$, with $L$ the coset representative of $\mathrm{SL}(3) / \mathrm{SO}(3)$ in a solvable basis

$$
L=\left(\begin{array}{ccc}
\mathrm{e}^{\frac{1}{2} \sigma_{1}+\frac{1}{2 \sqrt{3}} \sigma_{2}} & \mathrm{e}^{-\frac{1}{2} \sigma_{1}+\frac{1}{2 \sqrt{3}} \sigma_{2}} \chi_{1} & \mathrm{e}^{-\frac{1}{\sqrt{3}} \sigma_{2}}\left(\chi_{1} \chi_{2}+\chi_{3}\right) \\
0 & \mathrm{e}^{-\frac{1}{2} \sigma_{1}+\frac{1}{2 \sqrt{3}} \sigma_{2}} & \mathrm{e}^{-\frac{1}{\sqrt{3}} \sigma_{2}} \chi_{2} \\
0 & 0 & \mathrm{e}^{-\frac{1}{\sqrt{3}} \sigma_{2}}
\end{array}\right) .
$$

Note that $\operatorname{det}(M)=1$. The five scalars, $\sigma_{1}, \sigma_{2}, \chi_{1}, \chi_{2}$ and $\chi_{3}$ are canonically normalised and together with the volume modulus $v$ we have six metric scalars as announced previously. The on-shell values $\sigma_{1}=\sigma_{2}=\chi_{i}=0$ give the "round sphere", which corresponds to our solution.

The $C_{3}$ field gives one canonically normalised axionic scalar $a$ via $C_{3}=a \epsilon_{3}$. The $B$-field and $C_{1}$ field both give rise to three canonically normalised scalars $b^{i}$ and $c_{i}$ as follows

$$
\begin{aligned}
B & =\frac{1}{2} b^{i} \epsilon_{i j k} e^{j} \wedge e^{k}, \\
C_{1} & =c_{i} e^{i} .
\end{aligned}
$$


The scalar potential gets contributions from the internal curvature, $V_{R}$, the $H$-flux, $V_{H}$, the RR p-form fluxes, $V_{p}$, and the source tension, $V_{D 6}$. We explicitly find

$$
\begin{aligned}
V_{R} & =\mathrm{e}^{(7 \alpha+\beta) v} q^{2}\left(-\frac{1}{2}(\operatorname{Tr}[M])^{2}+\operatorname{Tr}\left[M^{2}\right]\right), \\
V_{0} & =\frac{1}{2} \mathrm{e}^{\frac{5}{2} \phi+(7 \alpha+3 \beta) v} F_{0}^{2}, \\
V_{D 6} & =\mathrm{e}^{\frac{3}{4} \phi+7 \alpha v} T_{D 6}, \\
V_{H} & =\frac{1}{2} h^{2} \mathrm{e}^{-\phi+(7 \alpha-3 \beta) v} \\
V_{2} & =\frac{1}{2} \mathrm{e}^{\frac{3}{2} \phi+(7 \alpha-\beta) v}\left(-q c_{i} \delta^{m i}+F_{0} b^{m}\right)\left(-q c_{j} \delta^{n j}+F_{0} b^{n}\right) M_{m n},
\end{aligned}
$$

where we have taken the $H$-field to be given by

$$
H=h \epsilon_{3}+\mathrm{d} B,
$$

with $B$ as above. In order for the $\mathrm{d} B$ term to generate a mass term for the $b^{i}$-scalars we need $\mathrm{d} B \sim \epsilon_{3}$, which cannot be done given the cohomology of $\mathrm{SU}(2) . F_{4}$ is not contributing any energy and hence, $a$ is a massless axion.

Our AdS solution stabilises the combinations $F_{0} b^{m}-q c_{i} \delta^{m i}$ while the orthogonal combinations remain flat. This allows us to set $b^{i}=c_{i}=0$. Also, since the three-sphere is round, we have $M=\mathbb{1}$, or, equivalently, $\sigma_{1}=\sigma_{2}=\chi_{1}=\chi_{2}=\chi_{3}=0$ at the vacuum. We also choose the dilaton, $\phi$, the axion $a$ and the volume scalar $v$ to be zero. Then all scalars are zero at the solution and we are at the origin of the scalar field space, where the metric is canonically normalised. These field values imply that we have to take the fluxes and tension appropriately

$$
F_{0}^{2}=\frac{4}{25} h^{2}, \quad T=\frac{2}{5} h^{2}, \quad q^{2}=\frac{28}{25} h^{2} .
$$

One can easily check that the origin in field space (i.e. all scalars zero) is an extremum of the potential.

Mathematica finds the following eigenvalues of $\left.\partial_{i} \partial_{j} V\right|_{\phi^{i}=0}$ at the origin of field space

$$
\lambda=h^{2}\left\{\frac{56}{25}, \frac{32}{25}, \frac{2}{25}(18 \pm \sqrt{79}), 0\right\}
$$

where the multiplicity of $\lambda=\frac{56}{25} h^{2}$ is 5 , the multiplicity of $\lambda=\frac{32}{25} h^{2}$ is 3 and the multiplicity of $\lambda=0$ is 4 . The other two eigenvalues have multiplicity equal to 1 . Since there are no negative mass modes the solution is stable with respect to these 14 left-invariant degrees of freedom.

\subsection{Open string moduli}

We have not taken into account the open string moduli since this is technically more challenging. The appropriate technique for this would be to construct the corresponding half-maximal gauged supergravity in $D=7$ since the gauged supergravity also contains 
the open string fields. We leave this for further research, but already comment on what one could expect on general grounds.

First of all one naively expects the moduli associated with the brane position to be massless as the round sphere is homogenous and hence without preferred points. The same is true for our fluxes that fill the sphere. However, there is the possibility of brane-flux annihilation, which can be a perturbative effect in some cases [25]. Note that the fluxes behave as $D 6$-branes when the sources are pure anti- $D 6$-branes and vice versa. Since the amount of fluxes equals the amount of brane charge the flux/brane annihilation process should be perturbative, at least this was the case for geometries dual to cascading gauge theories in [25]. It seems sensible that this applies to our situation as well. Brane/flux annihilation should occur via the Myers effect [26], which implies the nucleation of a D8brane, wrapping a 2 -sphere inside the 3 -sphere. However, this presumed instability would be absent for the solution with just a single $D 6$-brane. It would be interesting to study the decay further and give a quantitative description. However, in this paper we want to understand the relation between smeared and localised sources, which we take to be a closed string problem.

\section{Localisation}

In [3] an intuitive argument was presented explaining why it is difficult to find static configurations composed of mutually non-BPS components. A simple example is an antibrane sitting in a background of ISD flux. What will happen in general is that the flux is attracted to and sucked up by the brane, and possibly annihilated. The equations that capture this behaviour in our setup are primarily the $F_{2}$ Bianchi identity and the equation of motion for the $H$ field. The Bianchi identity determines $F_{2}$ in terms of the sources, including $F_{0} H$, and the equation of motion for $H$ determines how $H$ responds to $F_{2}$. A combination of both equations provides a very powerful tool to restrict possible configurations.

In the following we will treat two cases, first we consider the case of delta-function sources and find strong constraints that rule out a large class of configurations. Then we focus on extended and fully regularised source profiles. We analyse the full system using a Taylor expansion, where we find some surprisingly strong analytical constraints on what kind of configurations are possible. Our results show that the fully smeared solution is the only regular solution to the equations of motion.

\subsection{The ansatz}

As a starting point we assume that all sources are located on the north or/and south pole of the three-sphere such that the setup preserves an $\mathrm{SO}(3)$-symmetry of the full $\mathrm{SO}(4)$ symmetry of the smeared solution. Although the backreaction might bring us away from the three-sphere, we see no physical reason for why it should break the symmetry exhibited by the setup. We can then assume that for any setup preserving these isometries one can find a metric which at most has the physical singularities (i.e. the sources) at the poles. 
The most general form of such a metric is

$$
\mathrm{d} s_{10}^{2}=\mathrm{e}^{2 A(\theta)} \mathrm{d} s_{7}^{2}+\mathrm{e}^{2 B(\theta)}\left(\mathrm{d} \theta^{2}+\mathrm{e}^{2 C(\theta)} \sin ^{2}(\theta) \mathrm{d} \Omega_{2}^{2}\right),
$$

where the second conformal factor $C$ can be absorbed into $B$ by a suitable coordinate transformation $\theta \rightarrow \tilde{\theta}(\theta)$ that keeps the sources at the poles. One can show that, if the original metric does not have any singularities away from the poles, then the same is true for the new metric for which $C$ is transformed away. Hence, without loss of generality, we can write

$$
\mathrm{d} s_{10}^{2}=\mathrm{e}^{2 A(\theta)} \mathrm{d} s_{7}^{2}+\mathrm{e}^{2 B(\theta)}\left(\mathrm{d} \theta^{2}+\sin ^{2}(\theta) \mathrm{d} \Omega_{2}^{2}\right),
$$

and assume $A, B$ to be regular away from the poles.

The flux ansatz is

$$
\begin{aligned}
H & =\lambda F_{0} \mathrm{e}^{\frac{7}{4} \phi} \star_{3} 1, \\
F_{2} & =\mathrm{e}^{-\frac{3}{2} \phi-7 A} \star_{3} \mathrm{~d} \alpha,
\end{aligned}
$$

where $\phi, \lambda$ and $\alpha$ are now functions depending on $\theta$ and we take $F_{0}$ to be constant. This is the most general ansatz compatible with the form equations of motion (2.4) and (2.6) and our symmetries. In our previous work [3], we have considered a similar ansatz for the $\mathrm{AdS}_{4} \times S^{3} \times S^{3}$ solution, but did not take into account a non-trivial profile for the variable $\lambda$. We then found an obstacle to localisation. Allowing the variable $\lambda$ is the most general ansatz and we should be able to fully settle the discussion on whether localisation is possible or not.

Hence, the problem is reduced to finding a set of five unknown functions $A, B, \phi, \lambda, \alpha$ depending on $\theta$ and obeying coupled second-order differential equations, which we now derive. A prime' denotes the derivative with respect to $\theta$, e.g., $A^{\prime}=\mathrm{d} A / \mathrm{d} \theta$.

The $F_{2}$-Bianchi identity implies

$$
\frac{\left(\mathrm{e}^{-\frac{3}{2} \phi-7 A+B} \sin ^{2} \theta \alpha^{\prime}\right)^{\prime}}{\mathrm{e}^{3 B} \sin ^{2} \theta}=\mathrm{e}^{\frac{7}{4} \phi} \lambda F_{0}^{2}+Q \delta(D 6),
$$

where $Q \delta(D 6)$ symbolizes all source contributions at the north and south pole and is therefore generically a sum of delta-functions. The $H$ equation of motion allows us to eliminate $\alpha$ in terms of $\lambda$

$$
\alpha=\mathrm{e}^{\frac{3}{4} \phi+7 A} \lambda+\alpha_{0}
$$

The dilaton equation gives

$$
\frac{\left(\mathrm{e}^{7 A+B} \sin ^{2} \theta \phi^{\prime}\right)^{\prime}}{\mathrm{e}^{7 A+3 B} \sin ^{2} \theta}=\mathrm{e}^{\frac{5}{2} \phi} F_{0}^{2}\left(\frac{5}{4}-\frac{\lambda^{2}}{2}\right)+\frac{3}{4} \mathrm{e}^{-14 A-2 B-\frac{3}{2} \phi}\left(\alpha^{\prime}\right)^{2}+\frac{3}{4} \mathrm{e}^{\frac{3}{4} \phi} T \delta(D 6),
$$

where $T \delta(D 6)$ symbolizes the sum over all localised tension contributions. The trace of the external Einstein equation gives

$$
\begin{aligned}
\frac{16}{7} R_{7} & =-96 \mathrm{e}^{-2 A}-16 \mathrm{e}^{-2 B}\left(7\left(A^{\prime}\right)^{2}+A^{\prime} B^{\prime}+\frac{\left(\sin ^{2} \theta A^{\prime}\right)^{\prime}}{\sin ^{2} \theta}\right) \\
& =\mathrm{e}^{\frac{5}{2} \phi} F_{0}^{2}\left(1-2 \lambda^{2}\right)-\mathrm{e}^{-14 A-2 B-\frac{3}{2} \phi}\left(\alpha^{\prime}\right)^{2}-\mathrm{e}^{\frac{3}{4} \phi} T \delta(D 6) .
\end{aligned}
$$


The internal Einstein equation is more involved. We first display the Ricci tensor

$$
\begin{aligned}
R_{\theta \theta} & =2-\frac{\left(\sin ^{2} \theta B^{\prime}\right)^{\prime}}{\sin ^{2} \theta}-7\left(A^{\prime}\right)^{2}-B^{\prime \prime}-7 A^{\prime \prime}+7 A^{\prime} B^{\prime}, \\
R_{\varphi \varphi} & =\mathrm{e}^{-2 B} g_{\varphi \varphi}\left(2-\left(B^{\prime}\right)^{2}-\frac{\left(\sin ^{2} \theta B^{\prime}\right)^{\prime}}{\sin ^{2} \theta}-\cot \theta(B+7 A)^{\prime}-7 A^{\prime} B^{\prime}\right),
\end{aligned}
$$

where $\varphi$ indicates the two remaining angles. The Einstein equation in the $(\theta \theta)$-direction leads to

$$
\begin{aligned}
& 2-\frac{\left(\sin ^{2} \theta B^{\prime}\right)^{\prime}}{\sin ^{2} \theta}-7\left(A^{\prime}\right)^{2}-B^{\prime \prime}-7 A^{\prime \prime}+7 A^{\prime} B^{\prime}= \\
& \quad \frac{1}{2}\left(\phi^{\prime}\right)^{2}+\frac{1}{16} \mathrm{e}^{\frac{5}{2} \phi+2 B} F_{0}^{2}\left(1+6 \lambda^{2}\right)-\frac{1}{16} \mathrm{e}^{-14 A-\frac{3}{2} \phi}\left(\alpha^{\prime}\right)^{2}+\frac{7}{16} \mathrm{e}^{\frac{3}{4} \phi+2 B} T \delta(D 6),
\end{aligned}
$$

whereas the Einstein equations in the $(\varphi \varphi)$-directions lead to

$$
\begin{aligned}
& 2-\left(B^{\prime}\right)^{2}-\frac{\left(\sin ^{2} \theta B^{\prime}\right)^{\prime}}{\sin ^{2} \theta}-\cot \theta(B+7 A)^{\prime}-7 A^{\prime} B^{\prime}= \\
& \frac{1}{16} \mathrm{e}^{\frac{5}{2} \phi+2 B} F_{0}^{2}\left(1+6 \lambda^{2}\right)+\frac{7}{16} \mathrm{e}^{-14 A-\frac{3}{2} \phi}\left(\alpha^{\prime}\right)^{2}+\frac{7}{16} \mathrm{e}^{\frac{3}{4} \phi+2 B} T \delta(D 6) .
\end{aligned}
$$

\subsection{Delta-function sources}

In the case of delta-function sources our tool is the combination of the $H$ equation of motion and the Bianchi identity for $F_{2}$. At any point away from the sources we can combine (4.5) and (4.6) by substituting $\lambda$ to find

$$
\frac{\left(\mathrm{e}^{-\frac{3}{2} \phi-7 A+B} \sin ^{2} \theta\right)^{\prime}}{\mathrm{e}^{3 B} \sin ^{2} \theta} \alpha^{\prime}+\mathrm{e}^{-\frac{3}{2} \phi-7 A-2 B} \alpha^{\prime \prime}=\alpha \mathrm{e}^{\phi-7 A} F_{0}^{2},
$$

where we have used that through a shift in $\alpha$ we can always set $\alpha_{0}=0$.

This equation tells us one important fact: for non-singular $\mathrm{e}^{\phi}, \mathrm{e}^{A}, \mathrm{e}^{B}$, the function $\alpha$ must obey at any extremum $\left(\alpha^{\prime}=0\right)$

$$
\operatorname{sgn} \alpha^{\prime \prime}=\operatorname{sgn} \alpha .
$$

Note that this is also true at either of the poles $(\theta=0, \pi)$, if there are no localised sources at that pole. To be able to make use of this equation, we need to know the behaviour of $\alpha$ close to the (anti-)brane. This brings us to the difficult discussion of what the boundary conditions should be, resemblant of the discussion of the boundary conditions for anti- $D 3$ branes at the tip of the conifold [27, 28].

BPS-like boundary conditions. Global tadpole cancellation requires the integrated flux to be of opposite BPS-type as the brane itself, which immediately raises concerns about the possible stability of the localised system. On the other hand, it is only the integrated flux that is fixed by tadpole cancellation, so one may hope that perhaps a judicious distribution of the flux relative to the branes and a suitable choice of boundary conditions could result in a static solution. 


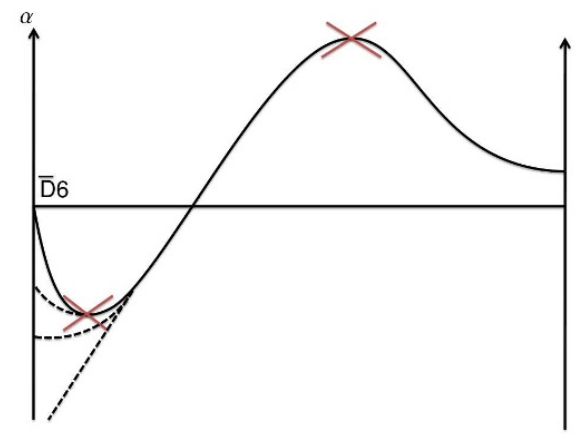

Figure 1. Configuration of a single $\overline{D 6}$-brane and flux. The solid line corresponds to the usual boundary condition of a BPS $\overline{D 6}$-brane, whereas the dashed lines represent more general boundary conditions consistent with net 'IASD' flux near the $\overline{D 6}$-brane. Global tadpole cancellation then enforces the forbidden extrema marked by the crosses.

The simplest possibility in this respect would be to take the boundary conditions as defined by the BPS D6-brane solution in massive IIA supergravity [29], which also involves H-flux and the Roman's mass $F_{0}$, as well as $F_{2}$-flux magnetically sourced by the brane. With these boundary conditions, the flux near the brane would be BPS with respect to the brane itself, and one would at least not expect instabilities to occur from the region close to the brane. As one moves away from the brane, the flux gradually has to change its BPS-type, as required by tadpole cancellation. Whether such a configuration has a chance to be also globally stable is not at all obvious. The interesting point now is that we actually do not need to know anything about the global stability of this flux setup, since we can rule it out completely using a simple 'topological' argument based on (4.14).

To see this, let us literally assume the standard BPS-boundary conditions at the (anti-) $D 6$-brane ${ }^{6}$ so that we have a near horizon region at the brane, where $\mathrm{e}^{A}$ and $\mathrm{e}^{\phi}$ approach zero, so that, in particular, $\mathrm{e}^{\frac{3}{4} \phi+7 A} \rightarrow 0$. An assumed ' $\mathrm{I}(\mathrm{A}) \mathrm{SD}$ ' flux ${ }^{7}$ near the (anti-) brane would mean $\lambda={ }_{{ }_{-}}^{+} 1$, and hence, using (4.6), $\alpha{ }_{(\gtrless)} 0$ for an (anti-)brane starting at $\alpha=0$. In figures 1 and 2, these standard BPS boundary conditions lead to the $\alpha$ profile indicated by the solid lines. If we do not insist on the flat space boundary conditions but still require a net 'I(A)SD' flux near the (anti-)brane, we would still have that $\alpha(\gtrless) 0$ near the brane, corresponding to the dashed lines in figure 1 and 2 .

The important point now is that, to ensure tadpole cancellation, the total integrated flux for (anti-)branes has to have exactly the opposite sign, $\int F_{0} H=F_{0}^{2} \int \alpha \mathrm{e}^{\phi-7 A} \star_{3} 1$ (§) 0 . Hence $\alpha$ must at some point change its sign. This generically requires an extremum with $\operatorname{sgn} \alpha^{\prime \prime}=-\operatorname{sgn} \alpha$, in contradiction with (4.14), so that with the BPS-like boundary con-

\footnotetext{
${ }^{6}$ The main differences of the BPS solutions [29] to our setup are the different world volume geometries (AdS vs. Minkowski), the compactness and curvature of the transverse dimensions and the different sign of the integrated flux $H F_{0}$. Zooming into the brane region with an assumed locally BPS flux, however, the large scale curvature and global structure should not matter.

${ }^{7}$ In lack of a better word, we will call the flux for $\lambda={ }_{(-)}^{+} 1$ that is mutually BPS with O6-planes and $D 6$-branes ( $\overline{O 6}$-planes and $\overline{D 6}$-branes) 'I(A)SD' and flux with $F_{0} H=\alpha F_{0}^{2} \mathrm{e}^{\phi-7 A} \star_{3} 1$ with $\alpha$ (之) 0 net 'I(A)SD'.
} 


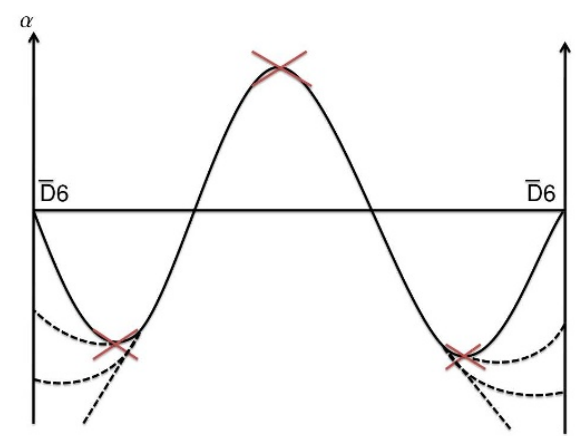

Figure 2. A configuration with a $\overline{D 6}$-brane at each pole with assumed 'IASD' flux near the branes, again leading to forbidden extrema.

ditions considered here, we can easily rule out certain combinations of fluxes and sources that are not mutually BPS.

Let us illustrate this with two examples. First consider the case of a single anti-D6brane located at $\theta=0$ and no source at $\theta=\pi$. This case is depicted in figure 1 .

Starting from the anti-brane, we have, close to the brane, $\alpha<0$, since the flux is locally net 'IASD'. To cancel the tadpole, the integrated flux must be positive, and hence $\alpha$ must become positive somewhere between the poles. Since we also have to have an extremum at the pole at $\theta=\pi$ due to rotational symmetry, $\alpha$ is forced to at least one extremum that violates (4.14) (marked by crosses in the figure). Note that, due to (4.14), the local maximum in figure 1 would also be excluded if it happens to be at the pole without the brane. We can also consider this case starting from the point $\theta=\pi$, where, as mentioned above, we have an extremum due to the rotational symmetry and the absence of a source. If, as is the case depicted in the figure, we start out at some positive $\alpha$ at that pole, $\alpha$ must grow. Then, to match with the boundary condition of the anti-brane at $\theta=0, \alpha$ has to have a maximum with $\alpha>0$, which is again forbidden by (4.14). If we start out at some negative $\alpha$ at $\theta=\pi, \alpha$ must decrease, and we could potentially match with the anti- $D 6$-brane at $\theta=0$. However, the net flux charge $\int H F_{0}$ would then be negative so that now the tadpole constraint would be violated. The case $\alpha(\pi)=0$ can be excluded in a similar manner.

In the second example, we place one anti-brane at $\theta=0$ and another at $\theta=\pi$. This situation is depicted in figure 2. Starting at $\theta=0$ the situation is just as before, we need $\alpha$ to flip sign to be able to cancel the tadpole, which leads to an extremum violating (4.14). In this case, coming from $\theta=\pi$ is just the mirrored situation. That is, it is not possible to match the 'IASD' boundary conditions of two anti-branes.

One might wonder how BPS setups constructed of anti-orientifold planes and 'IASD' fluxes fit in our discussion. Although the geometry is different in those cases, our qualitative argument can be applied similarly, since it does not rely on the details of the internal space. We show a setup with anti-O6-planes at each pole in figure 3.

The boundary conditions for the anti-O6-planes are different from the standard D6brane boundary conditions: instead of having $\mathrm{e}^{\frac{3}{4} \phi+7 A}$ zero, this factor diverges at the 


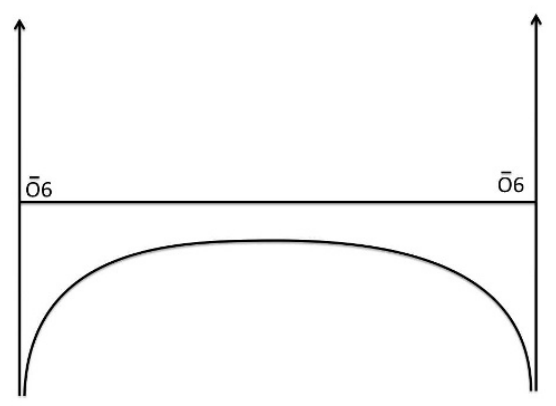

Figure 3. BPS configuration with orientifold-planes.

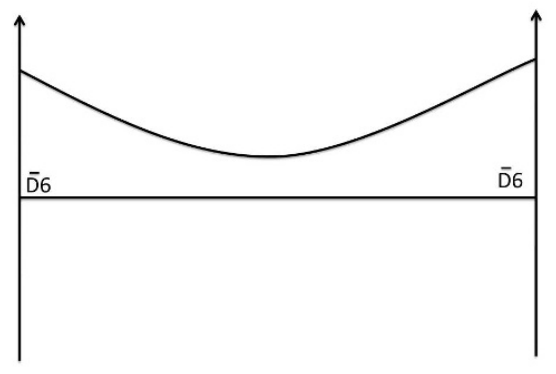

Figure 4. Alternative boundary conditions.

plane, hence $\alpha$ diverges. Furthermore, the anti-orientifold charge and the 'IASD' flux are already mutually BPS, and $\alpha$ never needs to switch sign to cancel the tadpole. This is the reason why the BPS situations survive localisation whereas the non-BPS situations collapse.

Alternative boundary conditions. Obviously one can draw a curve for $\alpha$, consistent with the global tadpole cancelation, that obeys (4.14) in its extrema. One example with an equal number of anti- $D 6$-branes at the north- and south-pole is given in figure 4 .

Note however that when the warp factor and the dilaton go to zero near the source, our argument rules out all net 'ISD' boundary conditions with finite $\lambda$. The only net 'ISD' cases we cannot rule out with this argument would then be such that $\lambda$ blows up at least as fast as the warp factor and dilaton go to zero so that $\alpha$ does not vanish (cf. (4.6)). Furthermore, the boundary conditions would have to be such that $\alpha^{\prime}<0$ at the anti-D6brane. It would be quite surprising if such solutions could be static, since the fluxes near the branes are not mutually BPS with the sources, so that one would expect net forces between the branes and the fluxes. It should be possible to check explicitly whether these anti-BPS-like boundary conditions could make any sense at all in our model, because the boundary conditions have to be consistent with the tension and charge multiplying the delta-function of the sources, which excludes many possible boundary behaviours. We will discuss this in more detail in [20]. 
In the rest of this paper, we instead give another argument against the localisability of the smeared solution presented in section 3, by viewing perfectly localised sources as a limiting case of regularised brane profiles of finite extent. This argument will be very general and does not use stability considerations or assumptions about boundary conditions and can hence be viewed as a complementary confirmation of the findings of the present subsection.

\subsection{Regularised sources}

We now explore the possibility of solutions with a regularised source profile, where the source is non-zero at points away from the poles. Let us consider extremal sources $|Q|=$ $T>0$ that have a regular profile with the source term written as

$$
T \delta(D 6)=z(\theta) \mathrm{e}^{-3 B}
$$

with $z(\theta)$ being a smooth function that becomes a constant in the smeared limit. The reason we took out the $\mathrm{e}^{-3 B}$ factor is that the delta-function, as it appears in the equations of motion, contains an inverse determinant of the metric.

Our setup has a consistent solution, if we can solve the equations of motion (4.5), (4.7), (4.8), (4.11), (4.12). We can check the consistency of these five differential equations by means of a Taylor expansion

$$
\begin{aligned}
\mathrm{e}^{2 A(\theta)} & =\sum_{n} a_{n}\left(\theta-\theta_{0}\right)^{n}, & \mathrm{e}^{2 B(\theta)} & =\sum_{n} b_{n}\left(\theta-\theta_{0}\right)^{n}, \\
\mathrm{e}^{\frac{1}{4} \phi(\theta)} & =\sum_{n} f_{n}\left(\theta-\theta_{0}\right)^{n}, & \lambda(\theta) & =\sum_{n} \lambda_{n}\left(\theta-\theta_{0}\right)^{n},
\end{aligned}
$$

around an arbitrary point $\left.\theta_{0} \in\right] 0, \pi\left[\right.$, where $z\left(\theta_{0}\right) \neq 0$. Plugging this expansion into the equations of motion, their coefficients $\sim\left(\theta-\theta_{0}\right)^{n}$ yield algebraic equations for every order $n$ that have to be satisfied at $\theta=\theta_{0}$. At every order $n$, one can then use the four equations (4.5), (4.7), (4.8), (4.11) to determine $a_{n+2}, b_{n+2}, f_{n+2}, \lambda_{n+2}$ in terms of the lowest order coefficients $q=\left\{a_{0}, b_{0}, f_{0}, \lambda_{0}, a_{1}, b_{1}, f_{1}, \lambda_{1}\right\}$. The remaining fifth equation (4.12) then gives a constraint between the elements of $q$ only. At zeroth order $(n=0)$, for example, $a_{2}, b_{2}, f_{2}, \lambda_{2}$ are determined in terms of $q$, whereas the fifth equation yields an additional constraint for $q$, and so on. Going to higher orders $n$, potentially produces an infinite amount of constraints between the elements of $q$ and thus should generically lead to strong constraints. $^{8}$

From the equations of the first six orders, we get the following conditions (upper sign for $D 6$-branes, lower sign for anti- $D 6$-branes as sources)

$$
a_{1}=f_{1}=\lambda_{1}=0, \quad \lambda_{0}=\mp \frac{5}{2}, \quad F_{0}^{2}=\frac{48}{7} \frac{1}{a_{0} f_{0}^{10}} .
$$

\footnotetext{
${ }^{8}$ This operation of finding constraints is very time consuming and sometimes, if not done in the correct order, too complex to solve on a desktop computer. Therefore the reader interested in verifying these computations should contact the authors to get more precise instructions.
} 


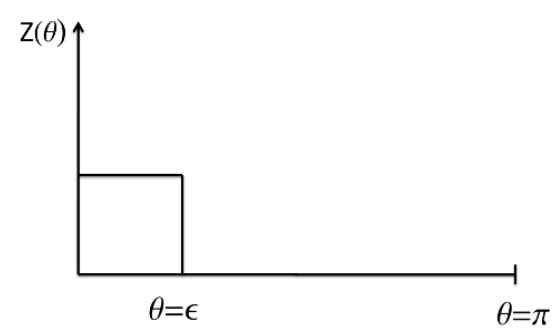

Figure 5. A brane source that is a step function.

Since $\theta_{0}$ was arbitrary these constraints must be satisfied for all $\left.\theta_{0} \in\right] 0, \pi[$, and hence

$$
\mathrm{e}^{2 A(\theta)}=a=\text { const. }, \quad \mathrm{e}^{\frac{1}{4} \phi(\theta)}=f=\text { const. }, \quad \lambda(\theta)=\mp \frac{5}{2}, \quad F_{0}^{2}=\frac{48}{7} \frac{1}{a f^{10}},
$$

everywhere (except at the poles). We can then plug this ansatz into the equations of motion (4.5), (4.7), (4.8), (4.11), (4.12) and solve for $B(\theta)$ and $z(\theta)$. We find that all equation are solved for

$$
\mathrm{e}^{2 B(\theta)}=\frac{4}{3}\left[\xi \cos (\theta)-\sqrt{\frac{16}{a}+\xi^{2}}\right]^{-2}, \quad z(\theta)=\frac{320}{7 \sqrt{3} a f^{3}}\left|\xi \cos (\theta)-\sqrt{\frac{16}{a}+\xi^{2}}\right|^{-3} .
$$

This solution has $z(\theta) \mathrm{e}^{-3 B}$ constant, which, with (4.15) and the fact that all fields except $B$ are constant, suggests that this is just the smeared solution written in a different coordinate system of the sphere. To find the corresponding coordinate transformation, consider

$$
\mathrm{d} s_{S^{3}}^{2}=\mathcal{R}_{S^{3}}^{2}\left(\mathrm{~d} \theta^{\prime 2}+\sin ^{2} \theta^{\prime} \mathrm{d} \Omega^{2}\right)=\mathrm{e}^{2 B(\theta)}\left(\mathrm{d} \theta^{2}+\sin ^{2} \theta \mathrm{d} \Omega^{2}\right) .
$$

Eliminating $\theta^{\prime}$ in terms of $\theta$ provides an ordinary differential equation for $\mathrm{e}^{2 B(\theta)}$ with solutions which agree exactly with (4.19). This means that we have proven that the smeared solution is the only solution to the equations of motion for which the source profile is a smooth function.

Note that our proof for obtaining the profile of $z(\theta)$ was valid in the support of $z(\theta)$. Hence one could still study the case for which, instead of an everywhere smooth function, the source profile is a step function as in figure 5 .

Then we should solve the vacuum equations outside of the source and match at the boundary $\theta=\epsilon$. For this to work the solution outside the source region should have a zero integrated total flux induced charge

$$
\int_{\epsilon}^{\pi} F_{0} H=0
$$

This follows because the regularised brane itself cancels exactly the flux-induced charge that is within the brane region $(\theta<\epsilon)$ so that the region outside the brane also has to have vanishing charge. Put differently, the source swallows as much flux within its spatial extent as it has charge. This implies that $\lambda$ is varying and switches sign outside the source. However, it is easy to prove that this cannot occur, using the same reasoning as before 
based on (4.14). The only thing we need is the behaviour of the fields at the boundary of the regularised source, where $\theta=\epsilon$. Assuming that the fields and their first derivatives are continuous at the step, we must have $\alpha^{\prime}=0$ (the same is true for the other fields), since otherwise we cannot consistently connect to the smeared solution inside the region $\theta<\epsilon$. Take for simplicity the solution which has positive $\alpha$ at the regularised source and $\alpha_{0}=0$ (the latter can be obtained by simply shifting $\alpha$ ). Then we have that, at $\theta=\epsilon$, (cf. (4.14))

$$
\alpha^{\prime \prime}>0 \text {. }
$$

So the function $\alpha$ is increasing when it leaves the brane. But we argued that it has to become negative in order to cancel the global tadpole. Therefore it has to reach a maximum somewhere. At the would-be maximum we have $\alpha^{\prime}=0$ and hence we would again find $\alpha^{\prime \prime}>0$ inconsistent with it being a maximum. Therefore $\alpha$, and thus $\lambda$, can never switch sign in order to cancel the global tadpole. The same happens when there are box-like brane sources at both poles.

In [3] we conjectured that a static non-BPS solution of the above kind could not be localised since the fluxes, for fixed $\lambda$, behave as smeared branes with the opposite charge of the real brane that cancels the tadpole. Therefore one expects the fluxes to be attracted into the localised branes. When the branes are smeared out this process does not occur since there is no preferred point of attraction. Rather one would expect brane-flux annihilation to take place but this is outside of the closed string sector we consider. One could have expected the no-go of [3] to be evaded by having a varying $\lambda$, in the same way that antibranes at the tip of the warped conifold, locally generate IASD fluxes [30], which, far away from the anti-branes change back into ISD fluxes. However, our above proof indicates that, when we do this for regularised branes, this is not possible and $\lambda$ remains constant, and hence the only solution is the fully smeared one.

\subsection{Summary of results}

In the case of fully localised sources we have shown, using "topological" arguments, that it is not possible to construct solutions, for a large class of boundary conditions. These arguments only use the Bianchi identity for $F_{2}$ and the $H$ equation of motion. The power of the argument is in its global nature. It could be that solutions exist locally around the north- and south-pole, but they are bound to collapse somewhere in the middle. Note that these arguments specifically use the choice of boundary conditions and the global tadpole cancellation condition. Hence in a non-compact situation (like the conifold) things might change. But non-compact models should be regarded as local models that eventually need an embedding in a global compact model for which the global tadpole indeed matters. The dependence on the boundary condition is also essential, and we have shown cases where there might be an option to evade our no-go for a given set of boundary conditions. However, if the fluxes near the source are mutually BPS with the source, then there is no solution.

To strengthen our results we have considered the possibility that the sources are not fully localised as a delta-function but instead have some remaining profile away from the 
pole on which the source is placed. Our results show that the solution is uniquely the fully smeared solution, which is not dependent on any assumptions of boundary conditions. The fact that there exists no source profile that is even a bit lumped around the poles makes it unlikely that solutions in the fully localised case exist.

This is in strong contrast with the BPS solutions given by the GKP backgrounds and their T-duals, since there one can find solutions to the equations of motion for an arbitrary source profile. The only fields that depend on the specific form of the source are the warp factor and the $F_{5}$-flux.

\section{Discussion}

One can regard the existence of flux vacua as a consequence of a balancing of various forces coming from localised sources, fluxes and curvature. When flux vacua are established using smeared sources, one has to make sure that the balancing of the forces does not rely on the sources being smeared out. If this is not satisfied, the solutions could not be trusted, since the balancing of the forces would be lost for the localised sources, and the solutions become non-static. Especially for non-BPS situations one might expect problems since one generically combines ingredients that are mutually non-BPS and therefore exert non-zero forces on each other. It is conceivable that smearing sources can undo such forces and give rise to fake solutions, which exist only due to the smearing. A simple example with mutually non-BPS ingredients is an anti-D3-brane in an ISD background, or its T-dual version with an anti- $D 6$-brane, considered in this paper in detail.

The localised version of such a smeared non-BPS solution would lead to brane-like sources surrounded by flux that, on the average, has the wrong sign to be mutually BPS with the source at a global level. However, if the flux changes its BPS-type near the branes so as to be mutually BPS with them in their vicinity, the balance of forces could be restored at least in the region near the branes. The question then is whether such a gradient in the flux type can be sustained and produce a static global solution.

We have investigated this using truly delta-like brane sources and found global obstructions for the existence of a solution, as long as arbitrarily close to the source the fluxes are mutually BPS with the sources. We furthermore investigated the existence of solutions with regularised brane sources and showed that the fully smeared solutions are unique, thereby confirming the conjecture made in [3]. The reason we investigated regularised sources as well is that they should tell us about what happens in the localised limit. Intuitively this can be understood by considering a truly localised brane whose profile is then a tiny bit smeared. We find in our setup that the entire background flux gets drawn into the brane region, which can be viewed as the physical reason for the complete breakdown of the solution upon localisation of the sources.

Our work is similar in spirit to the investigations of the backreaction of anti- $D 3$ branes in throat geometries (see e.g. [27, 28, 30-34] and references therein) but is different in the sense that we do not break supersymmetry perturbatively by just a few anti-branes. Instead we have as many SUSY-breaking branes as there is background flux. However, as we show in the appendix we can also find smeared solutions with a tunable amount of 
SUSY-breaking branes, such that we can be arbitrarily close to the BPS no-scale Minkowski solutions of $[1,3]$. We study the localisation of these solutions in [20], where we also discuss the boundary conditions at the sources in more detail.

Our results have particular relevance for attempts to find well-controlled de Sitter vacua at leading order in the $g_{s}$ and $\alpha^{\prime}$ expansion [35]. Such classical de Sitter compactifications are surprisingly difficult to engineer due to a number of simple no-go theorems. The simplest way around these no-go theorems seems to require the inclusion of orientifold planes in combination with compact spaces of negative integrated scalar curvature [36-38]. In the limit of smeared $O$-planes on a few negatively curved manifolds, classical de Sitter solutions were indeed identified in [39-44]. ${ }^{9}$ The known de Sitter models of this type are still not satisfactory, as they all have a tachyonic instability (i.e. they correspond to saddle points rather than local minima in the effective potential), and they suffer from reliability issues when flux quantization is taken into account [44]. However, a more basic issue with compactifications on negatively curved spaces was raised in [47]. There it was pointed out that in absence of warping and noticeable quantum corrections, negative curvature spaces require the presence of a continuous distribution of negative energy density as given by a smeared $O$-plane. Upon localisation of the $O$-planes, however, an everywhere negatively curved compact space can only be maintained when there are strong warping effects everywhere or the classical approximation breaks down. As the localisation of a smeared source does induce a nontrivial warp factor, one can potentially maintain negative internal curvature, and indeed in a BPS-like Minkowski configuration, one can explicitly show that this is possible [3]. The importance of our result lies in the observation that in non-BPS cases, such as de Sitter solutions, a localised solution may simply not exist after all. Note also that our results do not depend on the size of the compact space, which clearly shows that localisation effects in flux compactifications are important for arbitrarily large internal spaces.

It should be emphasised that our results not only cast doubts on the validity of classical de Sitter vacua that have been constructed in the limit of smeared sources, but that, more generally, non-BPS configurations supported by smeared $D$-branes or $O$-planes may not have reliable localized counterparts, even when they are perturbatively stable in the usual gauged supergravity truncation, involve AdS-spaces or compact spaces of positive curvature.

\section{Acknowledgments}

We like to thank Iosif Bena, Iñaki García Etxebarria, Ben Heidenreich, Fernando Marchesano, Liam McAllister, Paul McGuirck, Diederik Roest, Gary Shiu, Yoske Sumitomo and Bret Underwood for useful discussions. U.D. is supported by the Swedish Research Council (VR) and the Göran Gustafsson Foundation. D.J. and M.Z. are supported by the German Research Foundation (DFG) within the Emmy Noether Program (Grant number ZA 279/1-2) and the Cluster of Excellence "QUEST". T.V.R. is supported by the Göran

\footnotetext{
${ }^{9}$ These solutions correspond to critical points of lower-dimensional supergravity theories. But there also exist examples which breaks supersymmetry at the level of the compactification [45, 46].
} 
Gustafsson Foundation. T.W. is supported by the Alfred P. Sloan Foundation and by the NSF under grant PHY-0757868. This research was supported in part by the National Science Foundation under Grant No. NSF PHY05-51164. T.W. thanks the Hong Kong Institute for Advanced Study for hospitality.

\section{A Smeared non-BPS solutions for general $d$ and $\eta$}

Let us consider a combination of smeared $p$-brane sources that can be a general mixture of $D p$-, anti- $D p$-, $O p$ - and anti- $O p$-planes. This way we can get arbitrarily close to the BPS point by taking an arbitrarily small amount of anti-branes compared to the background $O$ planes. This makes sense in the supergravity picture where we do not bother about charge quantisation. Since the question we address is a supergravity question (the existence of localised solutions), we settle with this imprecise state of affairs. Because this mixture might be non-BPS we allow a resulting charge $Q$ and tension $T$ that might have different magnitudes, i.e.,

$$
T=\eta Q
$$

where $\eta$ measures how far we are from a BPS situation. For example $\eta=1$ with $Q<0$ means a BPS mixture of (anti-) $O p$ and (anti-) $D p$ sources such that the orientifold tension dominates. Or when $\eta=-1$ and $Q<0$ this corresponds to a net anti- $D p$ charge and tension. We furthermore point out that there also exist tachyonic de Sitter solutions with $\eta<-1$ when we allow $O p$ - and anti-Op-planes at the same time. It is not immediately clear to us whether this can be done consistently in the context of flux compactifications, but examples with $O p$ - and anti- $O p$-planes appeared in [48].

\section{A.1 The ansatz}

We look for solutions with constant dilaton, $\phi_{0}$, and an internal space that is a direct product of two spaces

$$
\begin{aligned}
\mathrm{d} s_{10}^{2} & =\mathrm{d} s_{p+1}^{2}+\mathrm{d} \Sigma_{3}^{2}+\mathrm{d} \Sigma_{6-p}^{2}, \\
H & =h \star_{3} 1, \\
F_{6-p} & =(-1)^{p} \mathrm{e}^{-\frac{p+1}{4} \phi_{0}} \kappa \star_{9-p} H,
\end{aligned}
$$

where $\mathrm{d} s_{p+1}^{2}$ is the metric of the external space, which we assume to be maximally symmetric (AdS, Mink, or dS), and $\mathrm{d} \Sigma_{3}^{2}$ and $\mathrm{d} \Sigma_{6-p}^{2}$ are metrics on Euclidean 3-, resp. $(6-p)$ dimensional Einstein spaces, sofar left unspecified. The parameter $\kappa$ is assumed constant. E.g., for $p=3$ we have that $\kappa=+1$ corresponds to ISD fluxes and $\kappa=-1$ to IASD fluxes. Since $H$ fills the $\Sigma_{3}$ space, the duality relation between $F_{6-p}$ and $H$ implies that $F_{6-p}$ fills the $\Sigma_{6-p}$ space, so these are truly simple ansätze.

Let us now go through all equations of motion. The $H$ and $F_{6-p}$ Bianchi identities are trivially satisfied whereas the $F_{8-p}$ Bianchi identity implies the tadpole condition ${ }^{10}$

$$
Q=-\mathrm{e}^{-\frac{p+1}{4} \phi_{0}} \kappa h^{2} .
$$

\footnotetext{
${ }^{10}$ In this appendix we set the volume of the internal space $V=1$ or equivalently take $Q$ and $T$ to denote the charge/tension densities.
} 
Furthermore, the $H, F_{6-p}$ and $F_{8-p}$ equations of motion are also satisfied. The dilaton equation, on the other hand implies a second-order polynomial for $\kappa$, which allows two solutions

$$
\kappa_{ \pm}=\frac{(p-3) \eta \pm \sqrt{(p-3)^{2} \eta^{2}+8(p-1)}}{2(p-1)} .
$$

This implies that we always have two branches of solutions corresponding to $\kappa_{+}$and $\kappa_{-}$.

Let us analyse the internal Einstein equation along the first space $\Sigma_{3}$. We find

$$
\left(R_{3}\right)_{a b}=\frac{\mathrm{e}^{-\phi_{0}}}{16} h^{2}\left(6-\kappa \eta(p+1)-(5-p) \kappa^{2}\right) g_{a b}^{(3)},
$$

which fixes the curvature of the Einstein space $\Sigma_{3}$. Similarly we find from the remaining directions in the internal Einstein equation

$$
\left(R_{6-p}\right)_{a b}=-\frac{\mathrm{e}^{-\phi_{0}}}{16} h^{2}\left(2+(p+1) \eta \kappa-(p+3) \kappa^{2}\right) g_{a b}^{(6-p)} .
$$

The external Einstein equation sets the value of the cosmological constant in the external dimensions

$$
R_{p+1}=-\frac{1}{8}(p+1) \mathrm{e}^{-\phi_{0}} h^{2}\left(1+\frac{5-p}{2} \kappa^{2}-\frac{(7-p)}{2} \eta \kappa\right) .
$$

Let us now analyse all the possible solutions, starting with recovering the BPS and non-BPS solutions of [3], where $\eta= \pm 1$.

\section{A.2 The solutions with $\eta= \pm 1$}

The solutions for $\kappa$ are (A.6)

$$
\eta= \pm 1 \quad \rightarrow \quad \kappa=\eta, \quad \kappa=-\eta \frac{2}{p-1} .
$$

From equation (A.5) this determines the sign of the charge and from that we find the sign of the tension using (A.1). For the "BPS" values, $\kappa=\eta= \pm 1$, we find that the tension is necessarily negative

$$
T(\kappa=\eta)=-h^{2} \mathrm{e}^{-\frac{p+1}{4} \phi_{0}},
$$

and that the solution is Minkowski and the internal spaces $\Sigma_{3}, \Sigma_{6-p}$ are flat. For $p=3$ this is the smeared GKP solution, built from O3-planes and ISD flux when $\eta=1$ and anti-O3-planes and IASD flux when $\eta=-1$.

For the non-BPS values $\kappa=-\frac{2}{p-1} \eta$ we find

$$
\begin{aligned}
R_{p+1} & =-\frac{(p+1)^{2}}{2(p-1)^{2}} \mathrm{e}^{-\phi_{0}} h^{2}, \\
\left(R_{3}\right)_{a b} & =\frac{(p+1)(p-2)}{2(p-1)^{2}} \mathrm{e}^{-\phi_{0}} h^{2} g_{a b}^{(3)}, \\
\left(R_{6-p}\right)_{a b} & =\frac{(p+1)}{2(p-1)^{2}} \mathrm{e}^{-\phi_{0}} h^{2} g_{a b}^{(6-p)}
\end{aligned}
$$


Hence these are AdS solutions with positively curved internal Einstein spaces, which we can for instance take to be spheres. ${ }^{11}$ Combining equations (A.1), (A.5) we find the total tension to be positive

$$
T\left(\kappa=\frac{-2}{p-1} \eta\right)=\frac{2}{p-1} h^{2} \mathrm{e}^{-\frac{p+1}{4} \phi_{0}},
$$

corresponding to net $D$-brane tension.

The solutions with $\eta= \pm 1$ are special in the sense that supersymmetry is not broken by the sources, but by choosing fluxes that are not mutually BPS with the branes. This implies that, under certain conditions, these AdS solutions are expected to be critical points of half-maximal gauged supergravities. This seems the case when we take as internal Einstein spaces the $S^{3} \times S^{6-p}$ (or $T^{3} \times S^{4}$ when $p=2$ ). This is because sphere-reductions, when consistent, are not expected to break the supersymmetry of the ten-dimensional action, only the sources break half of the supersymmetries. Especially for $p=3,5$ and 6 we are bound to have a description in terms of half maximal gauged supergravity since $S^{3} \times S^{6-p}$ is then a group manifold and dimensionally reducing on a group manifold is consistent [23].

\section{A.3 The solutions with general $\eta$}

Let us now consider general $\eta$. We find

$$
\begin{aligned}
R_{p+1} & =-\frac{(p+1)}{2(p-1)} \mathrm{e}^{-\phi_{0}} h^{2}(1-\eta \kappa), \\
\left(R_{3}\right)_{a b} & =\frac{(p-2)}{2(p-1)} \mathrm{e}^{-\phi_{0}} h^{2}(1-\eta \kappa) g_{a b}^{(3)}, \\
\left(R_{6-p}\right)_{a b} & =\frac{1}{2(p-1)} \mathrm{e}^{-\phi_{0}} h^{2}(1-\eta \kappa) g_{a b}^{(6-p)},
\end{aligned}
$$

with the total tension of the sources given by

$$
T=-\mathrm{e}^{-\frac{p+1}{4} \phi_{0}} \eta \kappa h^{2} .
$$

From these expressions we notice the following structure

- For the range $\kappa \eta<1$ we have non-BPS AdS vacua with positively curved internal Einstein spaces.

- For the range $\kappa \eta=1$ we have the BPS Minkowski solutions with flat internal spaces and net orientifold sources.

- For the range $\kappa \eta>1$ we have de Sitter solutions with negatively curved internal Einstein spaces and net orientifold sources.

By using the dependence of $\kappa$ on $\eta$ we can verify that $\kappa \eta>1$ necessarily implies $\eta^{2}>1$, which implies that the de Sitter solutions are only possible for combinations of $O p$ - and anti- $O p$-planes since such combinations have more negative tension than charge. The Minkowski turning point $\eta \kappa=1$ implies $\eta^{2}=1$ and is thus only possible for BPS sources.

\footnotetext{
${ }^{11}$ The case $p=2$ is exceptional because the 3 -space $\Sigma_{3}$ filled with $H$-flux is flat.
} 
In order to understand the perturbative stability of the solution we also computed the masses of 3 universal scalar fields: the dilaton $\phi$, the volume modulus $\varphi_{1}$, and the modulus $\varphi_{2}$ that measures the relative sizes of the two internal Einstein spaces (and does not exist for the $p=6$ solution). We found that the AdS solutions are stable within this subspace of degrees of freedom and the dS solutions are not.

\section{References}

[1] S.B. Giddings, S. Kachru and J. Polchinski, Hierarchies from fluxes in string compactifications, Phys. Rev. D 66 (2002) 106006 [hep-th/0105097] [SPIRES].

[2] M. Graña, R. Minasian, M. Petrini and A. Tomasiello, A scan for new $N=1$ vacua on twisted tori, JHEP 05 (2007) 031 [hep-th/0609124] [SPIRES].

[3] J. Blåbäck et al., Smeared versus localised sources in flux compactifications, JHEP 12 (2010) 043 [arXiv: 1009.1877] [SPIRES].

[4] C.P. Burgess and L. van Nierop, Bulk axions, brane back-reaction and fluxes, JHEP 02 (2011) 094 [arXiv: 1012.2638] [SPIRES].

[5] C.P. Burgess and L. van Nierop, Large dimensions and small curvatures from supersymmetric brane back-reaction, JHEP 04 (2011) 078 [arXiv: 1101.0152] [SPIRES].

[6] P. Koerber and D. Tsimpis, Supersymmetric sources, integrability and generalized-structure compactifications, JHEP 08 (2007) 082 [arXiv:0706.1244] [SPIRES].

[7] C. Caviezel et al., The effective theory of type IIA AdS 4 compactifications on nilmanifolds and cosets, Class. Quant. Grav. 26 (2009) 025014 [arXiv:0806.3458] [SPIRES].

[8] D. Cassani and A.-K. Kashani-Poor, Exploiting $N=2$ in consistent coset reductions of type IIA, Nucl. Phys. B 817 (2009) 25 [arXiv:0901.4251] [SPIRES].

[9] D. Roest, M-theory and gauged supergravities, Fortschr. Phys. 53 (2005) 119 [hep-th/0408175] [SPIRES].

[10] B.S. Acharya, F. Benini and R. Valandro, Fixing moduli in exact type IIA flux vacua, JHEP 02 (2007) 018 [hep-th/0607223] [SPIRES].

[11] P. Koerber, D. Lüst and D. Tsimpis, Type IIA AdS 4 compactifications on cosets, interpolations and domain walls, JHEP 07 (2008) 017 [arXiv:0804.0614] [SPIRES].

[12] S.B. Giddings and A. Maharana, Dynamics of warped compactifications and the shape of the warped landscape, Phys. Rev. D 73 (2006) 126003 [hep-th/0507158] [SPIRES].

[13] P. Koerber and L. Martucci, From ten to four and back again: how to generalize the geometry, JHEP 08 (2007) 059 [arXiv:0707.1038] [SPIRES].

[14] G. Shiu, G. Torroba, B. Underwood and M.R. Douglas, Dynamics of warped flux compactifications, JHEP 06 (2008) 024 [arXiv: 0803.3068] [SPIRES].

[15] A.R. Frey, G. Torroba, B. Underwood and M.R. Douglas, The universal Kähler modulus in warped compactifications, JHEP 01 (2009) 036 [arXiv:0810.5768] [SPIRES].

[16] L. Martucci, On moduli and effective theory of $N=1$ warped flux compactifications, JHEP 05 (2009) 027 [arXiv: 0902.4031] [SPIRES].

[17] M.R. Douglas, Effective potential and warp factor dynamics, JHEP 03 (2010) 071 [arXiv:0911.3378] [SPIRES]. 
[18] B. Underwood, A breathing mode for warped compactifications, arXiv:1009.4200 [SPIRES].

[19] D. Lüst, F. Marchesano, L. Martucci and D. Tsimpis, Generalized non-supersymmetric flux vacua, JHEP 11 (2008) 021 [arXiv:0807.4540] [SPIRES].

[20] J. Blåbäck et al., to appear.

[21] E. Silverstein, TASI/PiTP/ISS lectures on moduli and microphysics, hep-th/0405068 [SPIRES].

[22] P. Koerber, Lectures on generalized complex geometry for physicists, Fortschr. Phys. 59 (2011) 169 [arXiv:1006.1536] [SPIRES].

[23] J. Scherk and J.H. Schwarz, How to get masses from extra dimensions, Nucl. Phys. B 153 (1979) 61 [SPIRES].

[24] N. Alonso Alberca et al., Domain walls of $D=8$ gauged supergravities and their $D=11$ origin, JHEP 06 (2003) 038 [hep-th/0303113] [SPIRES].

[25] S. Kachru, J. Pearson and H.L. Verlinde, Brane/flux annihilation and the string dual of a non-supersymmetric field theory, JHEP 06 (2002) 021 [hep-th/0112197] [SPIRES].

[26] R.C. Myers, Dielectric-branes, JHEP 12 (1999) 022 [hep-th/9910053] [SPIRES].

[27] I. Bena, M. Graña and N. Halmagyi, On the existence of meta-stable vacua in Klebanov-Strassler, JHEP 09 (2010) 087 [arXiv:0912.3519] [SPIRES].

[28] P. McGuirk, G. Shiu and Y. Sumitomo, Non-supersymmetric infrared perturbations to the warped deformed conifold, Nucl. Phys. B 842 (2010) 383 [arXiv:0910.4581] [SPIRES].

[29] B. Janssen, P. Meessen and T. Ortín, The D8-brane tied up: string and brane solutions in massive type IIA supergravity, Phys. Lett. B 453 (1999) 229 [hep-th/9901078] [SPIRES].

[30] O. DeWolfe, S. Kachru and M. Mulligan, A gravity dual of metastable dynamical supersymmetry breaking, Phys. Rev. D 77 (2008) 065011 [arXiv:0801.1520] [SPIRES].

[31] I. Bena, G. Giecold and N. Halmagyi, The backreaction of anti-M2 branes on a warped Stenzel space, JHEP 04 (2011) 120 [arXiv:1011.2195] [SPIRES].

[32] D. Baumann, A. Dymarsky, S. Kachru, I.R. Klebanov and L. McAllister, D3-brane potentials from fluxes in AdS/CFT, JHEP 06 (2010) 072 [arXiv:1001.5028] [SPIRES].

[33] A. Dymarsky, On gravity dual of a metastable vacuum in Klebanov-Strassler theory, JHEP 05 (2011) 053 [arXiv: 1102.1734] [SPIRES].

[34] I. Bena, G. Giecold, M. Graña, N. Halmagyi and S. Massai, On metastable vacua and the warped deformed conifold: analytic results, arXiv:1102.2403 [SPIRES].

[35] M.P. Hertzberg, S. Kachru, W. Taylor and M. Tegmark, Inflationary constraints on type IIA string theory, JHEP 12 (2007) 095 [arXiv:0711.2512] [SPIRES].

[36] E. Silverstein, Simple de Sitter solutions, Phys. Rev. D 77 (2008) 106006 [arXiv:0712.1196] [SPIRES].

[37] S.S. Haque, G. Shiu, B. Underwood and T. Van Riet, Minimal simple de Sitter solutions, Phys. Rev. D 79 (2009) 086005 [arXiv:0810.5328] [SPIRES].

[38] T. Wrase and M. Zagermann, On classical de Sitter vacua in string theory, Fortschr. Phys. 58 (2010) 906 [arXiv:1003.0029] [SPIRES]. 
[39] R. Flauger, S. Paban, D. Robbins and T. Wrase, Searching for slow-roll moduli inflation in massive type IIA supergravity with metric fluxes, Phys. Rev. D 79 (2009) 086011 [arXiv:0812.3886] [SPIRES].

[40] C. Caviezel et al., On the cosmology of type IIA compactifications on $\mathrm{SU}(3)$-structure manifolds, JHEP 04 (2009) 010 [arXiv:0812.3551] [SPIRES].

[41] U.H. Danielsson, S.S. Haque, G. Shiu and T. Van Riet, Towards classical de Sitter solutions in string theory, JHEP 09 (2009) 114 [arXiv: 0907. 2041] [SPIRES].

[42] C. Caviezel, T. Wrase and M. Zagermann, Moduli stabilization and cosmology of type IIB on $\mathrm{SU}(2)$-structure orientifolds, JHEP 04 (2010) 011 [arXiv:0912.3287] [SPIRES].

[43] U.H. Danielsson, P. Koerber and T. Van Riet, Universal de Sitter solutions at tree-level, JHEP 05 (2010) 090 [arXiv: 1003.3590] [SPIRES].

[44] U.H. Danielsson et al., De Sitter hunting in a classical landscape, arXiv:1103.4858 [SPIRES].

[45] X. Dong, B. Horn, E. Silverstein and G. Torroba, Micromanaging de Sitter holography, Class. Quant. Grav. 27 (2010) 245020 [arXiv: 1005.5403] [SPIRES].

[46] D. Andriot, E. Goi, R. Minasian and M. Petrini, Supersymmetry breaking branes on solvmanifolds and de Sitter vacua in string theory, JHEP 05 (2011) 028 [arXiv:1003.3774] [SPIRES].

[47] M.R. Douglas and R. Kallosh, Compactification on negatively curved manifolds, JHEP 06 (2010) 004 [arXiv: 1001.4008] [SPIRES].

[48] S. Kachru, J. Kumar and E. Silverstein, Orientifolds, RG flows and closed string tachyons, Class. Quant. Grav. 17 (2000) 1139 [hep-th/9907038] [SPIRES]. 